\title{
A LAPLACE DECOMPOSITION ALGORITHM APPLIED TO A CLASS OF NONLINEAR DIFFERENTIAL EQUATIONS
}

\author{
SUHEIL A. KHURI
}

Received 24 January 2001 and in revised form 22 June 2001

In this paper, a numerical Laplace transform algorithm which is based on the decomposition method is introduced for the approximate solution of a class of nonlinear differential equations. The technique is described and illustrated with some numerical examples. The results assert that this scheme is rapidly convergent and quite accurate by which it approximates the solution using only few terms of its iterative scheme.

\section{Introduction}

This paper presents a Laplace transform numerical scheme, based on the decomposition method, for solving nonlinear differential equations. The analysis will be adapted to the approximate solution of a class of nonlinear second-order initial-value problems, though the algorithm is well suited for a wide range of nonlinear problems. The numerical technique basically illustrates how the Laplace transform may be used to approximate the solution of the nonlinear differential equation by manipulating the decomposition method which was first introduced by Adomian [1, 2]. The underlying idea of the technique is to assume an infinite solution of the form $u=\sum_{n=0}^{\infty} u_{n}$, then apply Laplace transformation to the differential equation. The nonlinear term is then decomposed in terms of Adomian polynomials and an iterative algorithm is constructed for the determination of the $u_{n}^{\prime} s$ in a recursive manner. The method is implemented for three numerical examples and the numerical results show that the scheme approximates the exact solution with a high degree of accuracy using only few terms of the iterative scheme. The main thrust of this technique is that the solution which is expressed as an infinite series converges fast to exact solutions. 
142 Laplace decomposition algorithm

The balance in this paper is as follows. In Section 2, the Laplace transform decomposition method will be presented as it applies to a class of secondorder nonlinear equations. In Section 3, the algorithm is implemented for three numerical examples.

\section{Numerical Laplace transform method}

In this paper, a Laplace transform decomposition algorithm is implemented for the solution of the following class of second-order nonlinear initial-value problems

$$
\begin{gathered}
y^{\prime \prime}+a(x) y^{\prime}+b(x) y=f(y) \\
y(0)=\alpha, \quad y^{\prime}(0)=\beta
\end{gathered}
$$

Here $f(y)$ is a nonlinear operator and $a(x)$ and $b(x)$ are known functions in the underlying function space. The technique consists first of applying Laplace transformation (denoted throughout this paper by $\mathcal{L}$ ) to both sides of (2.1), hence

$$
\mathcal{L}\left[y^{\prime \prime}\right]+\mathcal{L}\left[a(x) y^{\prime}\right]+\mathcal{L}[b(x) y]=\mathcal{L}[f(y)]
$$

Applying the formulas on Laplace transform, we obtain

$$
s^{2} \mathcal{L}[y]-y(0) s-y^{\prime}(0)+\mathcal{L}\left[a(x) y^{\prime}\right]+\mathcal{L}[b(x) y]=\mathcal{L}[f(y)]
$$

Using the initial conditions (2.2), we have

$$
s^{2} \mathcal{L}[y]=\beta+\alpha s-\mathcal{L}\left[a(x) y^{\prime}\right]-\mathcal{L}[b(x) y]+\mathcal{L}[f(y)]
$$

or

$$
\mathcal{L}[y]=\frac{\alpha}{s}+\frac{\beta}{s^{2}}-\frac{1}{s^{2}} \mathcal{L}\left[a(x) y^{\prime}\right]-\frac{1}{s^{2}} \mathcal{L}[b(x) y]+\frac{1}{s^{2}} \mathcal{L}[f(y)]
$$

The Laplace transform decomposition technique consists next of representing the solution as an infinite series, namely,

$$
y=\sum_{n=0}^{\infty} y_{n}
$$

where the terms $y_{n}$ are to be recursively computed. Also the nonlinear operator $f(y)$ is decomposed as follows:

$$
f(y)=\sum_{n=0}^{\infty} A_{n},
$$


where $A_{n}=A_{n}\left(y_{0}, y_{1}, y_{2}, \ldots, y_{n}\right)$ are the so-called Adomian polynomials. The first few polynomials are given by

$$
\begin{aligned}
& A_{0}=f\left(y_{0}\right), \\
& A_{1}=y_{1} f^{(1)}\left(y_{0}\right), \\
& A_{2}=y_{2} f^{(1)}\left(y_{0}\right)+\frac{1}{2 !} y_{1}^{2} f^{(2)}\left(y_{0}\right), \\
& A_{3}=y_{3} f^{(1)}\left(y_{0}\right)+y_{1} y_{2} f^{(2)}\left(y_{0}\right)+\frac{1}{3 !} y_{1}^{3} f^{(3)}\left(y_{0}\right) .
\end{aligned}
$$

Substituting (2.7) and (2.8) into (2.6) results

$$
\begin{aligned}
\mathcal{L}\left[\sum_{n=0}^{\infty} y_{n}\right]= & \frac{\alpha}{s}+\frac{\beta}{s^{2}}-\frac{1}{s^{2}} \mathcal{L}\left[a(x) \sum_{n=0}^{\infty} y_{n}^{\prime}\right] \\
& -\frac{1}{s^{2}} \mathcal{L}\left[b(x) \sum_{n=0}^{\infty} y_{n}\right]+\frac{1}{s^{2}} \mathcal{L}\left[\sum_{n=0}^{\infty} A_{n}\right] .
\end{aligned}
$$

Using the linearity of Laplace transform it follows that

$$
\begin{aligned}
\sum_{n=0}^{\infty} \mathcal{L}\left[y_{n}\right]= & \frac{\alpha}{s}+\frac{\beta}{s^{2}}-\frac{1}{s^{2}} \sum_{n=0}^{\infty} \mathcal{L}\left[a(x) y_{n}^{\prime}\right] \\
& -\frac{1}{s^{2}} \sum_{n=0}^{\infty} \mathcal{L}\left[b(x) y_{n}\right]+\frac{1}{s^{2}} \sum_{n=0}^{\infty} \mathcal{L}\left[A_{n}\right] .
\end{aligned}
$$

Matching both sides of (2.11) yields the following iterative algorithm:

$$
\begin{aligned}
& \mathcal{L}\left[y_{0}\right]=\frac{\alpha}{s}+\frac{\beta}{s^{2}}, \\
& \mathcal{L}\left[y_{1}\right]=-\frac{1}{s^{2}} \mathcal{L}\left[a(x) y_{0}^{\prime}\right]-\frac{1}{s^{2}} \mathcal{L}\left[b(x) y_{0}\right]+\frac{1}{s^{2}} \mathcal{L}\left[A_{0}\right] \\
& \mathcal{L}\left[y_{2}\right]=-\frac{1}{s^{2}} \mathcal{L}\left[a(x) y_{1}^{\prime}\right]-\frac{1}{s^{2}} \mathcal{L}\left[b(x) y_{1}\right]+\frac{1}{s^{2}} \mathcal{L}\left[A_{1}\right] .
\end{aligned}
$$

In general,

$$
\mathcal{L}\left[y_{n+1}\right]=-\frac{1}{s^{2}} \mathcal{L}\left[a(x) y_{n}^{\prime}\right]-\frac{1}{s^{2}} \mathcal{L}\left[b(x) y_{n}\right]+\frac{1}{s^{2}} \mathcal{L}\left[A_{n}\right] .
$$

Applying the inverse Laplace transform to (2.12) we get

$$
y_{0}=\alpha+\beta x \text {. }
$$

Substituting this value of $y_{0}$ into (2.13) gives

$$
\mathcal{L}\left[y_{1}\right]=-\frac{1}{s^{2}} \mathcal{L}[\beta a(x)]-\frac{1}{s^{2}} \mathcal{L}[b(x)(\alpha+\beta x)]+\frac{1}{s^{2}} \mathcal{L}\left[A_{0}\right] .
$$


144 Laplace decomposition algorithm

Evaluating the Laplace transform of the quantities on the right-hand side of (2.17) then applying the inverse Laplace transform, we obtain the value of $y_{1}$. The other terms $y_{2}, y_{3}, \ldots$ can be obtained recursively in a similar fashion using (2.15).

\section{Numerical examples}

The Laplace transform decomposition algorithm, described in Section 2, is applied to some special cases of the class of nonlinear initial-value problems given in (2.1) and (2.2).

Example 3.1. Consider the nonlinear problem

$$
\begin{aligned}
& y^{\prime \prime}+(1-x) y^{\prime}-y=2 y^{3} \\
& y(0)=1, \quad y^{\prime}(0)=1
\end{aligned}
$$

whose closed form solution is

$$
y=\frac{1}{1-x}
$$

Taking Laplace transform of both sides of (3.1) gives

$$
s^{2} \mathcal{L}[y]-y(0) s-y^{\prime}(0)=-\mathcal{L}\left[(1-x) y^{\prime}\right]+\mathcal{L}[y]+2 \mathcal{L}\left[y^{3}\right]
$$

The initial conditions (3.2) imply

$$
s^{2} \mathcal{L}[y]=s+1-\mathcal{L}\left[(1-x) y^{\prime}\right]+\mathcal{L}[y]+2 \mathcal{L}\left[y^{3}\right]
$$

or

$$
\mathcal{L}[y]=\frac{1}{s}+\frac{1}{s^{2}}-\frac{1}{s^{2}} \mathcal{L}\left[(1-x) y^{\prime}\right]+\frac{1}{s^{2}} \mathcal{L}[y]+\frac{2}{s^{2}} \mathcal{L}\left[y^{3}\right] .
$$

Following the technique, if we assume an infinite series solution of the form (2.7) we obtain

$$
\begin{aligned}
\mathcal{L}\left[\sum_{n=0}^{\infty} y_{n}\right]= & \frac{1}{s}+\frac{1}{s^{2}}-\frac{1}{s^{2}} \mathcal{L}\left[(1-x) \sum_{n=0}^{\infty} y_{n}^{\prime}\right] \\
& +\frac{1}{s^{2}} \mathcal{L}\left[\sum_{n=0}^{\infty} y_{n}\right]+\frac{2}{s^{2}} \mathcal{L}\left[\sum_{n=0}^{\infty} A_{n}\right],
\end{aligned}
$$

where the nonlinear operator $f(y)=y^{3}$ is decomposed as in (2.8) in terms of the Adomian polynomials. From (2.9) the first few Adomian polynomials 
for $f(y)=y^{3}$ are given by

$$
\begin{aligned}
& A_{0}=y_{0}^{3}, \\
& A_{1}=3 y_{0}^{2} y_{1}, \\
& A_{2}=3 y_{0}^{2} y_{2}+3 y_{0} y_{1}^{2}, \\
& A_{3}=3 y_{0}^{2} y_{3}+6 y_{0} y_{1} y_{2}+y_{1}^{3}
\end{aligned}
$$

Upon using the linearity of Laplace transform then matching both sides of (3.7), results in the iterative scheme

$$
\begin{aligned}
& \mathcal{L}\left[y_{0}\right]=\frac{1}{s}+\frac{1}{s^{2}} \\
& \mathcal{L}\left[y_{1}\right]=-\frac{1}{s^{2}} \mathcal{L}\left[(1-x) y_{0}^{\prime}\right]+\frac{1}{s^{2}} \mathcal{L}\left[y_{0}\right]+\frac{2}{s^{2}} \mathcal{L}\left[A_{0}\right] \\
& \mathcal{L}\left[y_{2}\right]=-\frac{1}{s^{2}} \mathcal{L}\left[(1-x) y_{1}^{\prime}\right]+\frac{1}{s^{2}} \mathcal{L}\left[y_{1}\right]+\frac{2}{s^{2}} \mathcal{L}\left[A_{1}\right] .
\end{aligned}
$$

In general,

$$
\mathcal{L}\left[y_{n+1}\right]=-\frac{1}{s^{2}} \mathcal{L}\left[(1-x) y_{n}^{\prime}\right]+\frac{1}{s^{2}} \mathcal{L}\left[y_{n}\right]+\frac{2}{s^{2}} \mathcal{L}\left[A_{n}\right]
$$

Operating with Laplace inverse on both sides of (3.9) gives

$$
y_{0}=1+x
$$

Substituting this value of $y_{0}$ and that of $A_{0}=y_{0}^{3}$ given in (3.8) into (3.10), we get

$$
\mathcal{L}\left[y_{1}\right]=\frac{1}{s^{2}} \mathcal{L}[2 x]+\frac{1}{s^{2}} \mathcal{L}\left[(1+x)^{3}\right]
$$

so

$$
\mathcal{L}\left[y_{1}\right]=\frac{2}{s^{4}}+\frac{2}{s^{2}}\left[\frac{1}{s}+\frac{3}{s^{2}}+\frac{6}{s^{3}}+\frac{6}{s^{4}}\right]=\frac{2}{s^{3}}+\frac{8}{s^{4}}+\frac{12}{s^{5}}+\frac{12}{s^{6}} .
$$

The inverse Laplace transform applied to (3.15) yields

$$
y_{1}=x^{2}+\frac{4}{3} x^{3}+\frac{1}{2} x^{4}+\frac{1}{10} x^{5}
$$

Substituting (3.16) into (3.11) and using the value of $A_{1}$ given in (3.8) implies

$$
\begin{aligned}
\mathcal{L}\left[y_{2}\right]= & -\frac{1}{s^{2}} L\left[(1-x)\left(2 x+4 x^{2}+2 x^{3}+\frac{1}{2} x^{4}\right)\right] \\
& +\frac{1}{s^{2}} \mathcal{L}\left[x^{2}+\frac{4}{3} x^{3}+\frac{1}{2} x^{4}+\frac{1}{10} x^{5}\right]+\frac{2}{s^{2}} \mathcal{L}\left[3 y_{0}^{2} y_{1}\right]
\end{aligned}
$$


146 Laplace decomposition algorithm

Table 3.1. Error obtained using Laplace transform numerical algorithm with four iterations.

\begin{tabular}{lll}
\hline$x$ & Error & Relative error \\
\hline 0.1 & $1.7858 \times 10^{-9}$ & $1.6072 \times 10^{-9}$ \\
0.2 & $9.4926 \times 10^{-8}$ & $7.5941 \times 10^{-8}$ \\
0.3 & $1.0220 \times 10^{-6}$ & $7.1542 \times 10^{-7}$ \\
0.4 & $1.2033 \times 10^{-5}$ & $7.2195 \times 10^{-6}$ \\
0.5 & $2.3196 \times 10^{-4}$ & $1.1598 \times 10^{-4}$ \\
0.6 & $3.2598 \times 10^{-3}$ & $1.3039 \times 10^{-3}$ \\
0.7 & $3.2952 \times 10^{-2}$ & $9.8855 \times 10^{-3}$ \\
\hline
\end{tabular}

Simplifying the right-hand side of (3.17) then applying the inverse Laplace transform, we obtain

$$
y_{2}=-\frac{1}{3} x^{3}+\frac{5}{12} x^{4}+\frac{7}{6} x^{5}+\frac{9}{10} x^{6}+\frac{38}{105} x^{7}+\frac{3}{40} x^{8}+\frac{1}{120} x^{9}
$$

Higher iterates can be easily obtained using the computer algebra system Maple. For example,

$$
\begin{aligned}
y_{3}= & \frac{1}{12} x^{4}-\frac{1}{4} x^{5}+\frac{1}{40} x^{6}+\frac{4}{5} x^{7}+\frac{25}{24} x^{8} \\
& +\frac{361}{540} x^{9}+\frac{3233}{12600} x^{10}+\frac{29}{462} x^{11}+\frac{11}{1200} x^{12}+\frac{11}{15600} x^{13} \\
y_{4}= & -\frac{1}{60} x^{5}+\frac{13}{180} x^{6}-\frac{41}{280} x^{7}-\frac{1213}{6720} x^{8} \\
& +\frac{7}{18} x^{9}+\frac{9991}{10800} x^{10}+\frac{14603}{16632} x^{11}+\frac{832991}{1663200} x^{12} \\
& +\frac{2066429}{10810800} x^{13}+\frac{20101}{400400} x^{14}+\frac{8101}{900900} x^{15}+\frac{211}{208000} x^{16}+\frac{211}{3536000} x^{17}
\end{aligned}
$$

Therefore, the approximate solution is

$$
\begin{aligned}
y= & y_{0}+y_{1}+y_{2}+y_{3}+y_{4}+\cdots \\
= & 1+x+x^{2}+x^{3}+x^{4}+x^{5}+\frac{359}{360} x^{6}+\frac{853}{840} x^{7}+\frac{2097}{2240} x^{8} \\
& +\frac{1151}{1080} x^{9}+\frac{17867}{15120} x^{10}+\frac{15647}{16632} x^{11}+\frac{848237}{1663200} x^{12}+\frac{518513}{2702700} x^{13} \\
& +\frac{20101}{400400} x^{14}+\frac{8101}{900900} x^{15}+\frac{211}{208000} x^{16}+\frac{211}{3536000} x^{17}+\cdots
\end{aligned}
$$

Table 3.1 exhibits the results of the approximation using only four iterations of the Laplace transform decomposition technique. The table shows the absolute error, that is, the difference between the approximate solution given 
Table 3.2. Error obtained using the [5,5] Padé approximant of the infinite series solution obtained by the Laplace transform numerical algorithm using four iterations.

\begin{tabular}{lll}
\hline$x$ & Error & Relative error \\
\hline 0.5 & $1.041 \times 10^{-5}$ & $5.2075 \times 10^{-6}$ \\
1.5 & $6.417 \times 10^{-5}$ & $3.2083 \times 10^{-5}$ \\
3 & $1.596 \times 10^{-5}$ & $3.1920 \times 10^{-5}$ \\
5 & $1.111 \times 10^{-5}$ & $4.4470 \times 10^{-5}$ \\
7 & $9.705 \times 10^{-6}$ & $5.8230 \times 10^{-5}$ \\
9 & $9.036 \times 10^{-6}$ & $7.2290 \times 10^{-5}$ \\
10 & $8.819 \times 10^{-6}$ & $7.9372 \times 10^{-5}$ \\
20 & $7.935 \times 10^{-6}$ & $1.5076 \times 10^{-4}$ \\
50 & $7.469 \times 10^{-6}$ & $3.6596 \times 10^{-4}$ \\
100 & $7.322 \times 10^{-6}$ & $7.2494 \times 10^{-4}$ \\
\hline
\end{tabular}

in (3.22) and the exact solution in (3.3), as well as the relative error. In both cases the error is less than $1 \%$. Note that the error is small for small values of $x$ and the accuracy degrades heavily for $x$ greater than 1 . The infinite series solution diverges for values of $x$ greater than 1, however we can use Maple to calculate the [5,5] Padé approximant of the infinite series solution (3.21) which gives the following rational fraction approximation to the solution:

$$
y \simeq \frac{1+\frac{53}{7} x+\frac{8703}{392} x^{2}-\frac{46607}{2058} x^{3}-\frac{178320109}{460992} x^{4}+\frac{1}{360} x^{5}}{1+\frac{46}{7} x+\frac{5735}{392} x^{2}-\frac{369191}{8232} \chi^{3}-\frac{55960047}{153664} x^{4}+\frac{2674820843}{6914880} x^{5}}
$$

In Table 3.2 we calculate the absolute and relative errors using this $[5,5]$ Padé approximant of the infinite series solution obtained by the Laplace decomposition algorithm. In both cases the error is less than $0.75 \%$. Clearly for large values of $x$, calculating the errors using the Pade approximant instead of the approximate infinite solution will lead to a drastic improvement in the degree of accuracy. The infinite series solution does not provide a good approximation for substantial values of $x$, however replacing the partial sum of the infinite series solution with its Padé approximant yields a very accurate rational solution.

Example 3.2. Consider the initial-value problem

$$
\begin{gathered}
y^{\prime}+y^{2}=1, \\
y(0)=3,
\end{gathered}
$$


148 Laplace decomposition algorithm

whose closed form solution is

$$
y=-1+\frac{2}{1-.5 e^{-2 x}} .
$$

First, we apply Laplace transform to both sides of (3.23),

$$
s \mathcal{L}[y]-y(0)+\mathcal{L}\left[y^{2}\right]=\frac{1}{s} .
$$

The initial condition (3.24) gives

$$
\mathcal{L}[y]=\frac{3}{s}+\frac{1}{s^{2}}-\frac{1}{s} \mathcal{L}\left[y^{2}\right] .
$$

Assuming an infinite series solution of the form (2.7), we have

$$
\mathcal{L}\left[\sum_{n=0}^{\infty} y_{n}\right]=\frac{3}{s}+\frac{1}{s^{2}}-\frac{1}{s} \mathcal{L}\left[\sum_{n=0}^{\infty} A_{n}\right],
$$

where the nonlinear operator $f(y)=y^{2}$ is decomposed as in (2.8) in terms of the Adomian polynomials. From (2.9) the first few Adomian polynomials are

$$
\begin{aligned}
& A_{0}=y_{0}^{2}, \\
& A_{1}=2 y_{0} y_{1}, \\
& A_{2}=2 y_{0} y_{2}+y_{1}^{2}, \\
& A_{3}=2 y_{0} y_{3}+2 y_{1} y_{2}
\end{aligned}
$$

Following the Laplace transform decomposition method, if we match both sides of (3.27) we obtain the iterative scheme

$$
\begin{gathered}
\mathcal{L}\left[y_{0}\right]=\frac{3}{s}+\frac{1}{s^{2}}, \\
\mathcal{L}\left[y_{1}\right]=-\frac{1}{s} \mathcal{L}\left[A_{0}\right], \\
\mathcal{L}\left[y_{2}\right]=-\frac{1}{s} \mathcal{L}\left[A_{1}\right],
\end{gathered}
$$

and the general iterative step is

$$
\mathcal{L}\left[\mathrm{y}_{\mathrm{n}+1}\right]=-\frac{1}{\mathrm{~s}} \mathcal{L}\left[\mathrm{A}_{\mathrm{n}}\right] .
$$

The inverse Laplace transform applied to (3.30) results

$$
y_{0}=3+x .
$$


Substituting $y_{0}=3+x$ and $A_{0}=y_{0}^{2}$ given in (3.8) into (3.31), we obtain

$$
\begin{aligned}
\mathcal{L}\left[y_{1}\right] & =-\frac{1}{s} \mathcal{L}\left[y_{0}^{2}\right]=-\frac{1}{s} \mathcal{L}\left[(3+x)^{2}\right] \\
& =-\frac{1}{s}\left(\frac{9}{s}+\frac{6}{s^{2}}+\frac{2}{s^{3}}\right)=-\frac{9}{s^{2}}-\frac{6}{s^{3}}-\frac{2}{s^{4}} .
\end{aligned}
$$

Consequently,

$$
y_{1}=-9 x-3 x^{2}-\frac{1}{3} x^{3}
$$

Using this value of $y_{1}$ into (3.32) yields

$$
\mathcal{L}\left[y_{2}\right]=-\frac{1}{s} \mathcal{L}\left[2 y_{0} y_{1}\right]=-\frac{2}{s} \mathcal{L}\left[-27 x-18 x^{2}-4 x^{3}-\frac{1}{3} x^{4}\right]
$$

or

$$
\mathcal{L}\left[y_{2}\right]=\frac{54}{s^{3}}+\frac{72}{s^{4}}+\frac{48}{s^{5}}+\frac{16}{s^{6}}
$$

Hence,

$$
y_{2}=27 x^{2}+12 x^{3}+2 x^{4}+\frac{2}{15} x^{5}
$$

The following higher iterates are obtained using Maple:

$$
\begin{aligned}
& y_{3}=-81 x^{3}-45 x^{4}-\frac{51}{5} x^{5}-\frac{17}{15} x^{6}-\frac{17}{315} x^{7}, \\
& y_{4}=243 x^{4}+162 x^{5}+\frac{231}{5} x^{6}+\frac{248}{35} x^{7}+\frac{62}{105} x^{8}+\frac{62}{2835} x^{9} .
\end{aligned}
$$

The infinite series solution becomes, upon using six iterations,

$$
\begin{aligned}
y= & 3-8 x+24 x^{2}-\frac{208}{3} x^{3}+200 x^{4}-\frac{8656}{15} x^{5}+\frac{24976}{15} x^{6}+\frac{553339}{315} x^{7} \\
& +\frac{15550}{21} x^{8}+\frac{502784}{2835} x^{9}+\frac{125536}{4725} x^{10}+\frac{78362}{31185} x^{11}+\cdots
\end{aligned}
$$

The $[3,3]$ Padé approximant of the solution obtained in (3.42) is given by

$$
y \simeq \frac{3+x+(6 / 5) x^{2}+(1 / 15) x^{3}}{1+3 x+(2 / 5) x^{2}+(1 / 5) x^{3}} .
$$

Table 3.3 gives the absolute and relative errors of the infinite series approximation using six iterations of the Laplace transform decomposition technique. The error is less than $0.025 \%$. As in Example 3.1, it was noticed that for large values of $x$, replacing the infinite series solution (3.42) with its Padé approximant (3.43) will improve the error. 
Laplace decomposition algorithm

Table 3.3. Error obtained upon using six iterations of the Laplace transform decomposition algorithm.

\begin{tabular}{lll}
\hline$x$ & Error & Relative error \\
\hline 0.1 & $2.9849 \times 10^{-10}$ & $1.2509 \times 10^{-10}$ \\
0.2 & $2.4803 \times 10^{-8}$ & $1.2351 \times 10^{-8}$ \\
0.3 & $2.9356 \times 10^{-7}$ & $1.6714 \times 10^{-7}$ \\
0.4 & $1.5941 \times 10^{-6}$ & $1.0092 \times 10^{-6}$ \\
0.5 & $5.6963 \times 10^{-6}$ & $3.9263 \times 10^{-6}$ \\
0.6 & $1.5688 \times 10^{-5}$ & $1.1581 \times 10^{-5}$ \\
0.7 & $3.6180 \times 10^{-5}$ & $2.8237 \times 10^{-5}$ \\
0.8 & $7.3380 \times 10^{-5}$ & $5.9923 \times 10^{-5}$ \\
0.9 & $1.3503 \times 10^{-4}$ & $1.1441 \times 10^{-4}$ \\
1.0 & $2.3023 \times 10^{-4}$ & $2.0105 \times 10^{-4}$ \\
\hline
\end{tabular}

Example 3.3. Consider the following nonlinear problem:

$$
\begin{gathered}
y^{\prime}=4 y-y^{3} \\
y(0)=0.5
\end{gathered}
$$

The exact solution is

$$
y=2\left(\frac{e^{8 x}}{e^{8 x}+15}\right)^{1 / 2}
$$

Operating with Laplace transform on both sides of (3.44) results

$$
s \mathcal{L}[y]-y(0)=4 \mathcal{L}[y]-\mathcal{L}\left[y^{3}\right]
$$

Using the initial condition (3.45) then simplifying the resulting equation in (3.47), we obtain

$$
\mathcal{L}[y]=\frac{0.5}{\mathrm{~s}}+\frac{4}{\mathrm{~s}} \mathcal{L}[\mathrm{y}]-\frac{1}{\mathrm{~s}} \mathrm{~L}\left[\mathrm{y}^{3}\right]
$$

Assuming an infinite series solution as in (2.7) we have

$$
\mathcal{L}\left[\sum_{n=0}^{\infty} y_{n}\right]=\frac{0.5}{s}+\frac{4}{s} \mathcal{L}\left[\sum_{n=0}^{\infty} y_{n}\right]-\frac{1}{s} \mathcal{L}\left[\sum_{n=0}^{\infty} A_{n}\right]
$$

where the nonlinear operator $f(y)=y^{3}$ is decomposed as in (2.8) in terms of the Adomian polynomials, which for this case the first few are given in (3.8). Matching both sides of (3.49), the components of $y$ can be defined 
as follows:

$$
\begin{aligned}
& \mathcal{L}\left[y_{0}\right]=\frac{0.5}{s} \\
& \mathcal{L}\left[y_{1}\right]=\frac{4}{s} \mathcal{L}\left[y_{0}\right]-\frac{1}{s} \mathcal{L}\left[A_{0}\right] \\
& \mathcal{L}\left[y_{2}\right]=\frac{4}{s} \mathcal{L}\left[y_{1}\right]-\frac{1}{s} \mathcal{L}\left[A_{1}\right]
\end{aligned}
$$

and the general term is

$$
\mathcal{L}\left[y_{n+1}\right]=\frac{4}{s} \mathcal{L}\left[y_{n}\right]-\frac{1}{s} \mathcal{L}\left[A_{n}\right] .
$$

The terms $y_{n}$ can be obtained in a recursive manner. Taking the inverse Laplace transform of (3.50) gives

$$
y_{0}=0.5 \text {. }
$$

Substituting this value of $y_{0}$ into (3.51), and using that $A_{0}=y_{0}^{3}$ from (3.8), we obtain

$$
\mathcal{L}\left[\mathrm{y}_{1}\right]=\frac{2}{\mathrm{~s}^{2}}-\frac{1}{\mathrm{~s}} \mathcal{L}\left[(0.5)^{3}\right]=\frac{2}{\mathrm{~s}^{2}}-\frac{1}{8 \mathrm{~s}^{2}}
$$

It follows that

$$
y_{1}=1.875 x
$$

Using this value of $y_{1}$ into (3.52) gives

$$
\mathcal{L}\left[\mathrm{y}_{2}\right]=\frac{4}{\mathrm{~s}} \mathcal{L}[1.875 x]-\frac{1}{\mathrm{~s}} \mathcal{L}\left[3 \mathrm{y}_{0}^{2} \mathrm{y}_{1}\right]
$$

Consequently

$$
y_{2}=3.046875 x^{2}
$$

The next higher iterates are obtained using Maple,

$$
\begin{aligned}
& y_{3}=1.54296875 x^{3}, \\
& y_{4}=-4.678955079 x^{4} .
\end{aligned}
$$

The series solution is therefore

$$
\begin{aligned}
y= & 0.5+1.875 x+3.046875 x^{2}+1.54296875 x^{3} \\
& -4.678955079 x^{4}-13.98919678 x^{5}+\cdots .
\end{aligned}
$$

The $[4,4]$ Padé approximant of this approximate solution is

$$
y \simeq \frac{0.5+2.940330021 x+9.262070767 x^{2}+13.01811008 x^{3}+9.597056446 x^{4}}{1+2.130660041 x+4.440416382 x^{2}-6.684988403 x^{3}+19.98685852 x^{4}}
$$


Table 3.4. Error obtained using four iterations of the numerical algorithm.

\begin{tabular}{lll}
\hline$x$ & Error & Relative error \\
\hline 0.05 & $2.5873 \times 10^{-7}$ & $4.3013 \times 10^{-7}$ \\
0.10 & $1.5733 \times 10^{-5}$ & $2.1885 \times 10^{-5}$ \\
0.15 & $1.6370 \times 10^{-4}$ & $1.9226 \times 10^{-4}$ \\
0.20 & $7.9299 \times 10^{-4}$ & $7.9581 \times 10^{-4}$ \\
0.25 & $2.3856 \times 10^{-3}$ & $2.0763 \times 10^{-3}$ \\
0.30 & $4.8087 \times 10^{-3}$ & $3.6942 \times 10^{-3}$ \\
0.35 & $5.6245 \times 10^{-3}$ & $3.8888 \times 10^{-3}$ \\
0.40 & $2.3004 \times 10^{-3}$ & $1.4601 \times 10^{-3}$ \\
\hline
\end{tabular}

Table 3.4 shows that the absolute and relative errors of the approximation (3.61), using four iterations of the numerical technique, is less than $2 \%$. Again, as in the previous examples, the Padé approximant (3.61) of the solution (3.60) yields a better approximation of the exact solution for larger values of $x$.

Example 3.4. In this last example, the method is illustrated by considering the damped Duffing's equation

$$
\begin{gathered}
y^{\prime \prime}+k y^{\prime}=-y^{3}, \\
y(0)=\alpha, \quad y^{\prime}(0)=\beta,
\end{gathered}
$$

where $k$ is a positive constant. Applying Laplace transform to both sides of (3.62) we obtain

$$
s^{2} \mathcal{L}[y]-y(0) s-y^{\prime}(0)+k(s \mathcal{L}[y]-y(0))=-\mathcal{L}\left[y^{3}\right]
$$

Simplifying this equation and using the initial conditions (3.63) yields

$$
\left(s^{2}+k s\right) \mathcal{L}[y]=\alpha(s+k)+\beta-\mathcal{L}\left[y^{3}\right]
$$

or

$$
\mathcal{L}[y]=\alpha \frac{s+k}{s^{2}+k s}+\frac{\beta}{s^{2}+k s}-\frac{1}{s^{2}+k s} \mathcal{L}\left[y^{3}\right] .
$$

Assuming an infinite series solution of the form (2.7) we get

$$
\mathcal{L}\left[\sum_{n=0}^{\infty} y_{n}\right]=\alpha \frac{s+k}{s^{2}+k s}+\frac{\beta}{s^{2}+k s}-\frac{1}{s^{2}+k s} \mathcal{L}\left[\sum_{n=0}^{\infty} A_{n}\right],
$$

where the nonlinear operator $f(y)=y^{3}$ is decomposed in terms of the Adomian polynomials which for this case are given in (3.8). Upon using 
the linearity of Laplace transform then matching both sides of (3.67), results in the iterative algorithm

$$
\begin{aligned}
& \mathcal{L}\left[y_{0}\right]=\alpha \frac{s+k}{s^{2}+k s}+\frac{\beta}{s^{2}+k s}, \\
& \mathcal{L}\left[y_{1}\right]=-\frac{1}{s^{2}+k s} \mathcal{L}\left[A_{0}\right], \\
& \mathcal{L}\left[y_{2}\right]=-\frac{1}{s^{2}+k s} \mathcal{L}\left[A_{1}\right] .
\end{aligned}
$$

In general,

$$
\mathcal{L}\left[\mathrm{y}_{\mathrm{n}+1}\right]=-\frac{1}{\mathrm{~s}^{2}+\mathrm{ks}} \mathcal{L}\left[\mathrm{A}_{\mathrm{n}}\right]
$$

Consider the case where $\alpha=\beta=k=1$. Operating with Laplace inverse on both sides of (3.68) gives

$$
y_{0}=2-e^{-x} .
$$

Substituting this value of $y_{0}$ and that of $A_{0}=y_{0}^{3}$ given in (3.8) into (3.69), we obtain

$$
\mathcal{L}\left[y_{1}\right]=-\frac{1}{s^{2}+k s} \mathcal{L}\left[y_{0}^{3}\right]=-\frac{1}{s^{2}+k s} \mathcal{L}\left[\left(2-e^{-x}\right)^{3}\right]
$$

so

$$
\mathcal{L}\left[y_{1}\right]=\frac{8 / s-12 /(s+1)+6 /(s+2)-1 /(3+s)}{s^{2}+s} .
$$

The inverse Laplace transform applied to (3.74) yields

$$
y_{1}=-8 x+\frac{52}{3}-12 x e^{-x}-\frac{29}{2} e^{-x}-3 e^{-2 x}+\frac{1}{6} e^{-3 x}
$$

Substituting the value of $A_{1}$ given in (3.8) into (3.70) implies

$$
\mathcal{L}\left[\mathrm{y}_{2}\right]=-\frac{1}{\mathrm{~s}^{2}+\mathrm{ks}} \mathcal{L}\left[3 \mathrm{y}_{0}^{2} \mathrm{y}_{1}\right]
$$

Substituting the values of $y_{0}$ and $y_{1}$ given in (3.72) and (3.75) into (3.76) then applying the inverse Laplace transform, we obtain

$$
\begin{aligned}
y_{2}= & 48 x^{2}-304 x+\frac{37049}{60}-\left(24 x^{2}+430 x+\frac{10543}{24}\right) e^{-x} \\
& -(60 x+185) e^{-2 x}+\left(6 x+\frac{71}{12}\right) e^{-3 x}+\frac{11}{12} e^{-4 x}-\frac{1}{40} e^{-5 x}
\end{aligned}
$$


Table 3.5. Error that results from comparing the solution derived by the Laplace transform numerical algorithm with four iterations, and the numerical solution obtained using Maple.

\begin{tabular}{lll}
\hline$x$ & Error & Relative error \\
\hline 0.1 & $1.6482 \times 10^{-10}$ & $1.5123 \times 10^{-10}$ \\
0.2 & $3.7401 \times 10^{-10}$ & $3.2275 \times 10^{-10}$ \\
0.3 & $3.6991 \times 10^{-8}$ & $3.0661 \times 10^{-8}$ \\
0.4 & $7.8776 \times 10^{-7}$ & $6.3895 \times 10^{-7}$ \\
0.5 & $8.5726 \times 10^{-6}$ & $6.9187 \times 10^{-6}$ \\
0.6 & $6.0690 \times 10^{-5}$ & $4.9478 \times 10^{-5}$ \\
0.7 & $3.1769 \times 10^{-4}$ & $2.6522 \times 10^{-4}$ \\
0.8 & $1.3281 \times 10^{-3}$ & $1.1494 \times 10^{-3}$ \\
0.9 & $4.6628 \times 10^{-3}$ & $4.2292 \times 10^{-3}$ \\
1.0 & $1.4236 \times 10^{-2}$ & $1.3664 \times 10^{-2}$ \\
\hline
\end{tabular}

In a similar fashion, higher iterates are obtained using Maple. For example,

$$
\begin{aligned}
y_{3}= & -320 x^{3}+3616 x^{2}-\frac{246667}{15} x+\frac{184833613}{6300} \\
& +\left(32 x^{3}-796 x^{2}-\frac{545719}{30} x-\frac{71534779}{3600}\right) e^{-x} \\
& +\left(84 x^{2}+\frac{1055}{3} x+\frac{9103}{144}\right) e^{-3 x}-\left(\frac{3}{2} x+\frac{29}{400}\right) e^{-5 x} \\
& -\left(360 x^{2}+4308 x+\frac{385069}{40}\right) e^{-2 x}+\left(\frac{121}{3} x+\frac{863}{9}\right) e^{-4 x} \\
& +\frac{19}{5040} e^{-7 x}-\frac{373}{1800} e^{-6 x} .
\end{aligned}
$$

Therefore, the approximate solution is

$$
\begin{aligned}
y= & y_{0}+y_{1}+y_{2}+y_{3}+\cdots \\
= & -\frac{251347}{15} x-320 x^{3}-\left(\frac{392589}{40}+4368 x+360 x^{2}\right) e^{-2 x} \\
& -\left(\frac{39}{400}+\frac{3}{2} x\right) e^{-5 x}+\left(84 x^{2}+\frac{1073}{3} x+\frac{9979}{144}\right) e^{-3 x} \\
& -\left(\frac{73172029}{3600}+32 x^{3}+\frac{558979}{30} x+820 x^{2}\right) e^{-x}+3664 x^{2} \\
& +\left(\frac{121}{3} x+\frac{3485}{36}\right) e^{-4 x}-\frac{373}{1800} e^{-6 x}+\frac{19}{5040} e^{-7 x}+\frac{94422779}{3150}+\cdots
\end{aligned}
$$


Table 3.5 shows the absolute and relative errors that result from comparing the approximate solution obtained from the Laplace transform decomposition algorithm using four iterations, and the numerical solution of the damped Duffing's equation evaluated using Maple solve commands. The error is less than $0.001 \%$. In all the previous four examples, it was observed that increasing the number of iterates will improve the accuracy of the solution.

\section{References}

[1] G. Adomian, A review of the decomposition method and some recent results for nonlinear equations, Comput. Math. Appl. 21 (1991), no. 5, 101-127. MR 92h:00002b. Zbl 0732.35003.

[2] Solving Frontier Problems of Physics: The Decomposition Method, Kluwer, Dordrecht, 1994. MR 95e:00026. Zbl 0802.65122.

Suheil A. Khuri: Department of Computer Science, Mathematics and Statistics, American University of Sharjah, United Arab Emirates

E-mail address: skhoury@aus.ac.ae 


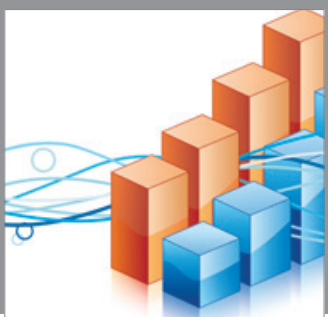

Advances in

Operations Research

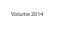

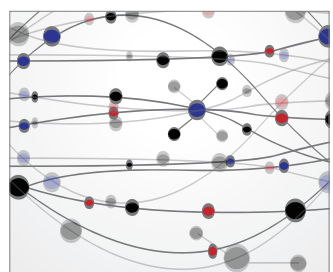

\section{The Scientific} World Journal
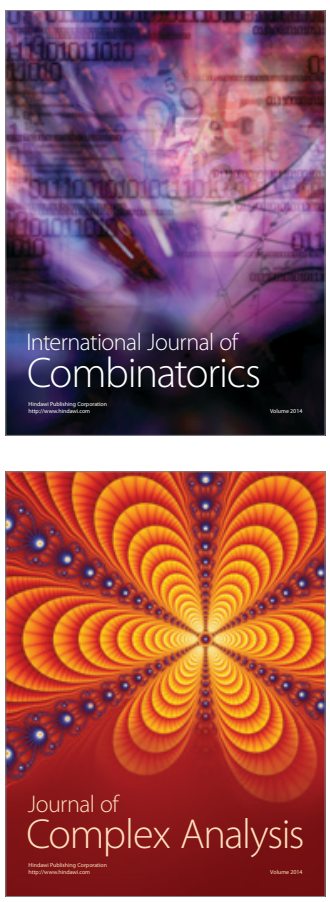

International Journal of

Mathematics and

Mathematical

Sciences
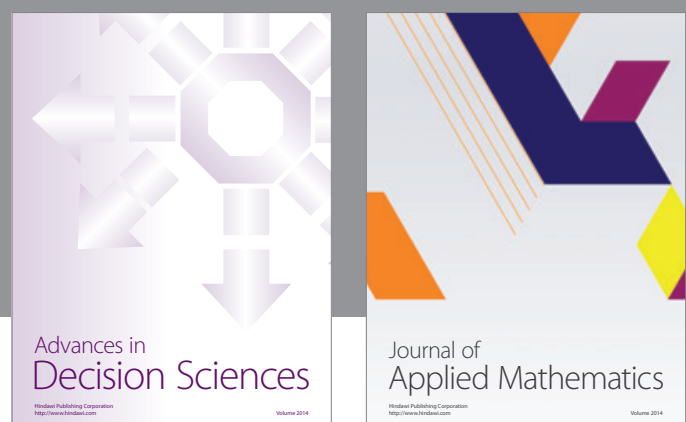

Journal of

Applied Mathematics
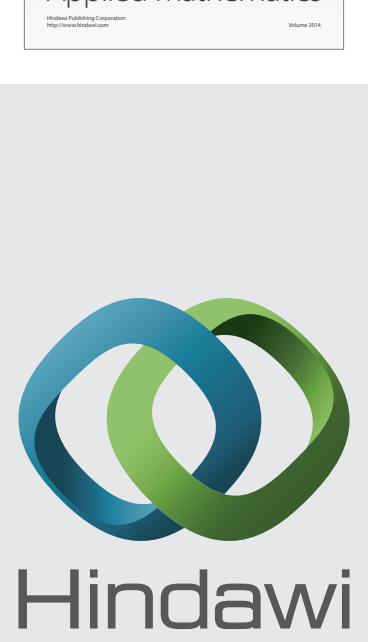

Submit your manuscripts at http://www.hindawi.com
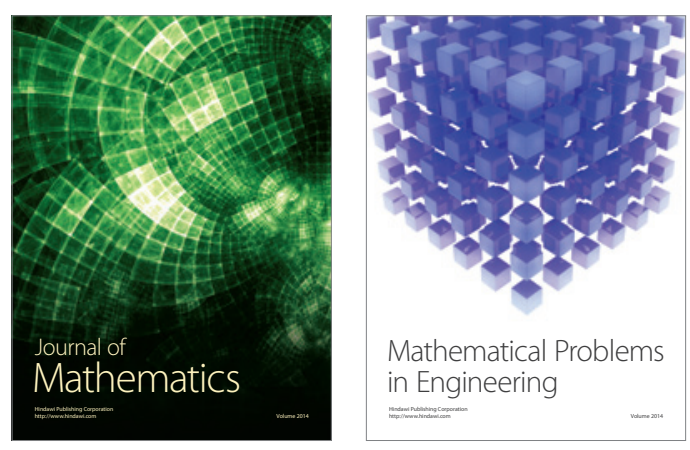

Mathematical Problems in Engineering
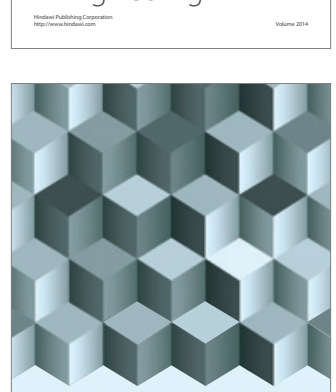

Journal of

Function Spaces
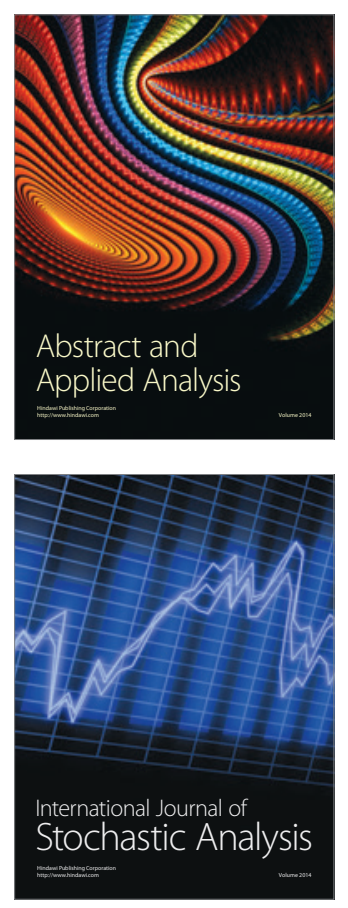

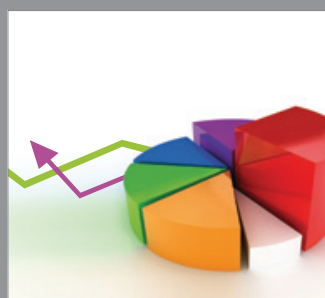

ournal of

Probability and Statistics

Promensencen
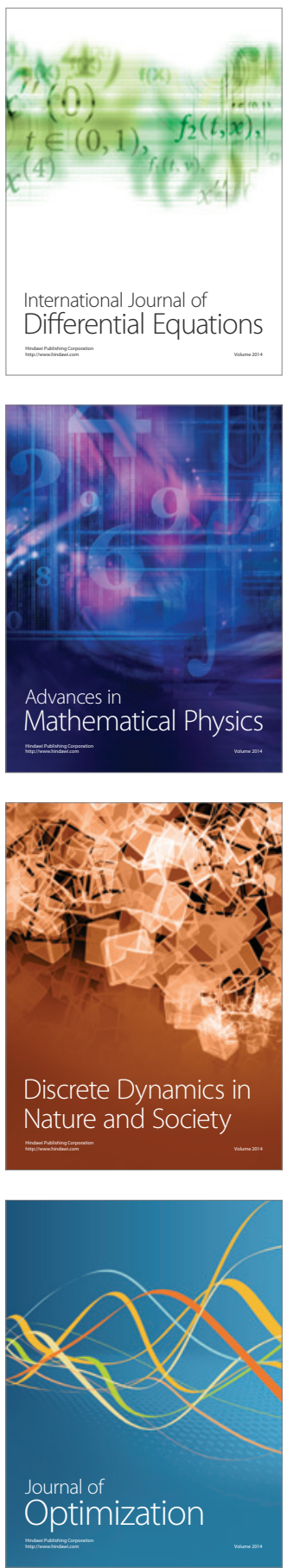